\title{
Clasificación, estructura y diversidad del matorRal SUB- MONTANO ADYACENTE A LA PLANICIE COSTERA DEL GOLFO Norte en el NORESTE de MÉXICO
}

\author{
Eduardo Estrada-Castillón ${ }^{1,3}$, José A. Villarreal-Quintanilla², Enrique Jurado-Ybarra, \\ César Cantú-Ayala ${ }^{1}$, Mario Alberto García-Aranda ${ }^{1}$, Jaime Sánchez-Salas ${ }^{1}$, \\ JaVier JIMÉNeZ-PÉrez Y y Marisela PANDo-Moreno ${ }^{1}$
}

${ }^{1}$ Facultad de Ciencias Forestales, Universidad Autónoma de Nuevo León ${ }^{2}$ Departamento de Botánica, Universidad Autónoma Agraria Antonio Narro

${ }^{3}$ Autor para la correspondencia: aeduardoestradac@prodigy.net.mx

\begin{abstract}
Resumen: Se estudió el matorral submontano adyacente a la Llanura Costera del Golfo en el estado de Nuevo León. Se registraron los valores de cobertura, densidad, altura, frecuencia y valor de importancia de todas las arbustivas presentes en los 39 sitios de muestreo. Se registraron 233 taxa, que incluyen 55 familias, 150 géneros y 228 especies de plantas vasculares. Del total de especies, 39 de ellas definen cerca del $95 \%$ de las diferentes asociaciones vegetales del matorral submontano. Se registran siete formas de crecimiento dominantes, arbustos, árboles, rosetófilas, suculentas, hierbas, enredaderas y parásitas. Los arbustos son los dominantes con 116 especies, seguidas por árboles (39) y hierbas suculentas (26). Los arbustos inermes son las especies predominantes en los tres estratos del matorral. Con base en valores de importancia de las 39 especies más importantes se clasificó el matorral submontano mediante análisis de conglomerados. Se reconocen cuatro grupos principales y ocho asociaciones de plantas. Se registraron 19 especies endémicas presentes en el matorral submontano, 15 hierbas, tres crasicaules (cactáceas pequeñas) y un árbol, no hay especies arbustivas endémicas en el matorral submontano. La principal causa de pérdida de matorral submontano en Nuevo León es su tala para desarrollo de asentamientos humanos y se da principalmente en la región metropolitana.

Palabras clave: análisis de conglomerados, conservación, crasicaules, formas de crecimiento, Nuevo León, México.
\end{abstract}

\begin{abstract}
A study of piedmont scrub adjacent to the Gulf Coastal Plain in the State Nuevo Leon was carried out. Canopy cover values, density, height, frequency, and importance value from all shrub species of the piedmont scrub were recorded in each of the 39 sites sampled. We recorded 233 taxa, including 55 families, 150 genera, and 228 species. Thirty nine of the species define almost $95 \%$ of the different vegetation associations of the piedmont scrub. Seven dominant growth forms were recorded, shrubs, trees, rosetophyllous, succulents, herbs, lianas, and parasites. The shrubs are the dominant specie (116 species), followed by trees (39) and shrubby and herbaceous succulents (26). Non-thorny shrubs are the dominant species in the three strata of the piedmont scrub. Based on importance values of the 39 most important species, piedmont scrub was classified by means of cluster analysis. Four main groups and eight plant associations are recognized. We recorded 19 endemic species into the piedmont scrub, 15 herbs, three succulents (small cacti) and one tree, there is not endemic shrubby species in the piedmont scrub. Logging is the main cause of piedmont scrub loss around metropolitan area: Logged areas are converted to human settlements.
\end{abstract}

Key words: cluster analysis, conservation, crasicaule, growth form, Nuevo Leon, Mexico.

L os matorrales son las comunidades vegetales más abundantes en México, cubriendo casi el $40 \%$ de su superficie, 800,000 $\mathrm{km}^{2}$ (Rzedowski, 1978). La palabra "piedmontscrub" fue acuñada por Muller (1939) para definir una comunidad vegetal generalmente caducifolia por un corto período de tiempo, el cual incluye arbustos altos o árboles bajos de origen neotropical. La clasificación y nomenclatura del matorral submontano ha cambiado a través del tiempo de acuerdo con los criterios de diferentes autores, destacando aquellos de tipo fisonómico (White, 1940; Miranda y Hernández-X., 1963; Moya-Rodríguez, 1982; RamírezÁlvarez, 1984; Alanís, 2004), composición vegetal (Muller, 1947; Villegas-Durán, 1972; Panti-Madero, 1992) y estructura (Rojas-Mendoza, 1965; Gutiérrez-Lobatos, 1970; Gon- 
zález-Medrano, 1972; Melgoza-Castillo, 1977; VillarrealQuintanilla, 1979; Briones-Villarreal, 1991; Panti-Madero, 1992; Challenger, 1998; Briones y Villarreal-Quintanilla, 2001; Alanís, 2004). Sin embargo, hay un acuerdo con respecto a su distribución, donde se destaca como una franja de vegetación localizada en la parte media y en las faldas de las principales cadenas montañosas de la Sierra Madre Oriental y en varias montañas aisladas en la región norte; así como con respecto a la heterogeneidad de su estructura (tres estratos principales) y su diversidad vegetal. En la actualidad, la clasificación de la vegetación de México, incluye al matorral submontano dentro del matorral xerófilo (Rzedowski, 1978).

El matorral submontano se desarrolla principalmente en climas, cálidos y semicálidos, con precipitaciones entre los 450 y los $900 \mathrm{~mm}$ anuales. Esta comunidad vegetal es de tipo subperennifolio con una combinación de elementos espinosos y no espinosos (INEGI, 1986a). La mayor parte del matorral submontano en el estado de Nuevo León se desarrolla sobre suelos someros, especialmente de origen sedimentario (Rojas-Mendoza, 1965; Rzedowski, 1966, 1978), aunque en ocasiones puede desarrollarse sobre estratos con suelos ígneos (Briones-Villarreal, 1991; Briones y Villarreal-Q, 2001), ocupa aproximadamente el 8\% (5,000 $\mathrm{km}^{2}$ ) del estado, desarrollándose principalmente entre los 450 y los 800 m (Muller, 1939; Rojas-Mendoza, 1965), rara vez hasta los 1,800 m (Rzedowski, 1978; Challenger, 1998). A pesar de su relativa homogeneidad fisonómica, el matorral submontano muestra una marcada heterogeneidad en su composición vegetal a través del gradiente latitudinal en el noreste de México.

En este trabajo se reconoce al matorral submontano con base en su distribución (parte media, laderas y base de las montañas) y su composición vegetal (cobertura, densidad, frecuencia y altura de las especies que lo constituyen). En Nuevo León, el matorral submontano sigue el mismo patrón (NE-SO) que el de las montañas de la región, a manera de un cinturón de vegetación de aproximadamente $270 \mathrm{~km}$ de largo. El límite sur de esta comunidad vegetal se encuentra en el estado de San Luis Potosí, donde parece ser una continuación del matorral submontano descrito para Guadalcazar (Rzedowski, 1966) y la Sierra de Catorce (González-Costilla et al., 2007; Giménez de Azcárate y González-Costilla, 2011) y su límite norte se encuentra en el municipio de Lampazos, Nuevo León (Marroquín et al., 1964; Rzedowski, 1978; Briones y Villarreal-Q, 2001).

La mayoría de las ciudades en el estado de Nuevo León (Monterrey, Montemorelos, Santiago, El Cercado, Linares, Allende, San Pedro Garza García, Santa Catarina, Guadalupe) se localizan o están rodeadas por el matorral submontano, principalmente en altitudes entre los 360 y 1,000 m. Durante los últimos 20 años, estas ciudades han incrementado considerablemente su crecimiento, con la consecuente remoción de extensas áreas de matorral submontano para la construcción de asentamientos humanos, particularmente en las porciones medias de las montañas, donde se presenta la mayor exuberancia y diversidad de especies de esta comunidad vegetal. Muchas personas del área rural del estado de Nuevo León basan parte de su economía en el aprovechamiento, manejo y uso del matorral submontano. Hay pequeñas fábricas de madera especializadas en carpintería, elaboración de carbón, artesanías de madera, elaboración de postería para cercas que se extraen de especies como: Condalia hookeri var. hookeri (brasil), Ebenopsis ebano (ébano), Gochnatia hypoleuca var. hypoleuca (ocotillo), Helietta parvifolia (barreta), Prosopis glandulosa var. torreyana (mezquite) y P. laevigata (mezquite) como fuente madera y carbón; Diospyros texana (chapote blanco), E. ebano (ébano), Havardia pallens(tenaza) para artículos de carpintería; y otras especies como Amyris madrensis (barretilla), Calliandra conferta (caliandra), Citharexylum berlandieri, Eysenhardtia texana (vara dulce) y Guaiacum angustifolium (guayacán) son utilizadas principalmente como forraje (Reid et al., 1990).

La mayoría de las aéreas utilizadas para caza (UMA's) en México se localizan en comunidades de matorral tamaulipeco y matorral submontano, las cuales albergan los más importantes trofeos para los cazadores tales como venado cola blanca, guajolote silvestre, jabalí y codorniz (SEMARNAT, 2007).

El objetivo de este trabajo fue clasificar las principales asociaciones vegetales del matorral submontano utilizando información cuantitativa de las especies, así como características de hábitat para proveer herramientas hacia un mejor manejo de esta comunidad vegetal, pero escasamente estudiada. Además, determinar las especies endémicas dentro de este tipo de vegetación y discutir el deterioro que ha tenido últimamente el matorral submontano y la importancia de las áreas de conservación de recursos naturales inmersas en ella.

\section{Métodos}

Sitio de estudio. El área de trabajo es un cinturón de vegetación que se distribuye a lo largo de la parte media, faldas y base de la cadena de montañas que constituyen la Sierra Madre Oriental, así como las sierras y serranías aisladas en su límite norte (Figura 1). Para los propósitos de este estudio, el límite sur de esta comunidad vegetal se registró en la parte sureste del municipio de Linares y su límite norte en el municipio de Lampazos (su distribución real en el norte del estado) entre las coordenadas geográficas: $24^{\circ} 33^{\prime}-27^{\circ} 13^{\prime}$ $\mathrm{N}$ y $99^{\circ} 20^{\prime}-100^{\circ} 50^{\prime} \mathrm{O}$.

Varias ciudades se localizan a lo largo de este cinturón de vegetación: Linares, Hualahuises, Montemorelos, Rayones, Allende, Congregación Calles, Lazarillos, Santiago, El Álamo, Monterrey, Sabinas Hidalgo, Salinas Victoria, Hidalgo, Villaldama, Bustamante y Lampazos, con una pobla- 


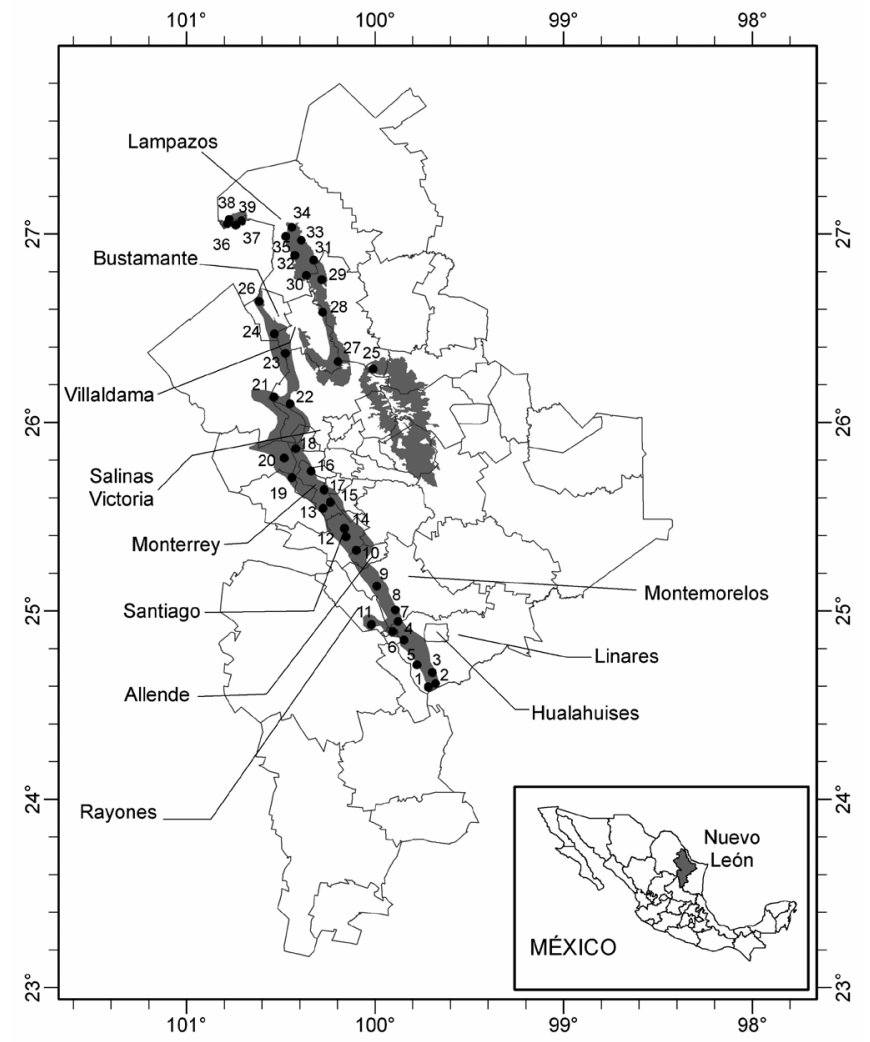

Figura 1. Estado de Nuevo León mostrando el área de distribución del matorral submontano y los 39 sitios de muestreo.

ción aproximada de 3,898,597 habitantes (INEGI, 1986c; CONABIO, 2010). Los suelos de esta área son ligeramente alcalinos, con pH entre 6.5 y 7.5, de color blanquecino, con diferentes profundidades de acuerdo con su ubicación en el relieve, pero generalmente entre 10 y $60 \mathrm{~cm}$ de profundidad, con textura limosa, arcillosa o arenosa-arcillosa. En las partes bajas de las montañas dentro del área de estudio se presentan materiales desintegrados de marga y pizarras que en ocasiones alcanzan varios metros de profundidad (Woerner, 1991; CONABIO, 2010). Las planicies, que en su límite altitudinal albergan matorral submontano, regularmente están constituidas por suelos aluviales y frecuentemente conglomerados, asociados con bosques de aciculifolios, escuamifolios, así como encinares (INEGI, 1986b, 2008).

Para el área de estudio se registran tres tipos de clima, el más húmedo, $(\mathrm{A}) \mathrm{C}(\mathrm{w} 1)(\mathrm{e}$ ')w”, semicálido-subhúmedo, se presenta en la porción media (municipios de Allende y Santiago), presenta lluvias en verano (hasta $1,020 \mathrm{~mm}$ de precipitación anuales) y una temperatura media entre los 18 y $22{ }^{\circ} \mathrm{C}$. En la porción sur (municipios de Montemorelos y Linares), el clima es semicálido-subhúmedo, (A)Cx'a(e), con lluvias en verano, pero con un período de sequía estival, con precipitaciones anuales entre los 805 y $980 \mathrm{~mm}$ y con temperatura medias entre los 22 y $22.3^{\circ} \mathrm{C}$, alcanzando los $40{ }^{\circ} \mathrm{C}$ o más en verano. La porción norte es la más árida, se localiza desde Monterrey hasta Lampazos, presenta clima de tipo BS, con dos subtipos. De Monterrey hasta Bustamante, predomina el subtipo semiseco-semicálido, BS1hw, con una temperatura media anual entre los 18 y $22{ }^{\circ} \mathrm{C}$ y una precipitación anual entre los 395 y $510 \mathrm{~mm}$ (García, 1973; CONABIO, 2010), mientras que en Lampazos se presenta el subtipo BSo(h')hx', seco-cálido, con una precipitación entre $\operatorname{los} 300$ y $500 \mathrm{~mm}$ y una temperatura media anual entre los 25 y los $28^{\circ} \mathrm{C}$.

Con base en mapas de edafología y vegetación, escala 1:150,000 (INEGI, 1986b), se localizaron 39 sitios de 1 ha a lo largo de la distribución del matorral submontano en el área de estudio (Figura 1). En cada sitio, se dispusieron al azar seis subparcelas de $100 \mathrm{~m}^{2}(10 \mathrm{~m} \times 10 \mathrm{~m})$ para cuantificar cobertura aérea, densidad, frecuencia y altura de las especies (Mueller-Dumbois y Ellenberg, 1974). En el centro de cada sitio se tomó una muestra de suelo para cuantificar textura, $\mathrm{pH}$, profundidad y contenido de material orgánica.

En este estudio, matorral bajo pertenece a asociaciones vegetales o plantas de $1 \mathrm{~m}$ de altura o menores, matorral mediano a aquellas entre $1.1 \mathrm{a} 2.5 \mathrm{~m}$ de altura y matorral alto a aquellas que crecen por encima de $2.6 \mathrm{~m}$ de altura; especies arbustivas espinosas son aquellas con espinas laterales o terminales; rosetófilas, aquellas especies con hojas en roseta basal (Agave, Dasylirion y Hesperaloe) o apical (Brahea, Sabal y Yucca) y arbustivas y hierbas suculentas, aquellas que ostentan tallos carnosos (cactáceas). Los ejemplares botánicos colectados se resguardan en la colección científica del herbario CFNL (Linares, Nuevo León).

Análisis de información. Los valores de las variables cuantificadas en las seis parcelas de cada cuadrante fueron promediados, los valores absolutos de cobertura, densidad y frecuencia de cada especie fueron transformados a valores relativos (Mueller-Dumbois y Ellenberg, 1974; Bonham, 1980) para calcular su valor de importancia, el cual fue utilizado para el análisis de conglomerados; todas las especies con valores de cobertura y densidad promedio de $0.1 \%$ o mayores y frecuencias relativas mayores de $5.1 \%$ fueron incluidas en el análisis estadístico. Se utilizó el Índice de similitud de Sörensen (Mueller-Dumbois y Ellenberg, 1974; Herrera, 1993) (Iss $=2 \mathrm{~W} /(\mathrm{A}+\mathrm{B}) \bullet 100)$, donde $\mathrm{W}$ es el valor de importancia de las especies en común en ambos sitios comparados, A son los valores de importancia las especies del sitio A y B son los valores de importancia las especies del sitio del sitio B. Se construyó una matriz de similitud para los 39 sitios (Digby y Kempton, 1991; Herrera, 1993). La nueva matriz con los índices de similitud fue analizada mediante el método de conglomerados, utilizando la técnica jerárquica politética aglomerativa (Gauch, 1982; Manly, 1992) y de varianza mínima (Ward, 1963) en el programa estadístico MVSP (Kovach Computing Services, 1998). Para estimar la distorsión de las distancias de la matriz original con respecto a la matriz resultante, el dendrograma 


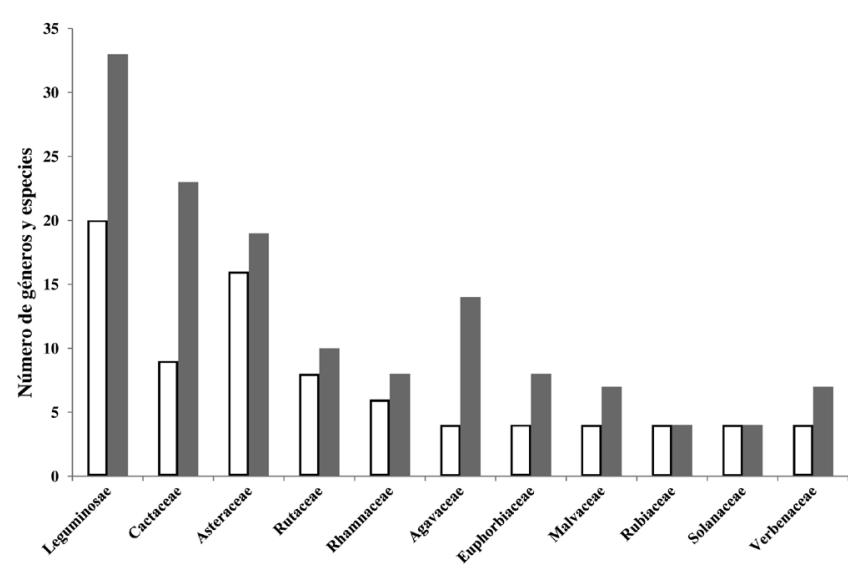

Figura 2. Familias con mayor número de géneros (blancos) y especies (grises) en el matorral submontano en el estado de Nuevo León, México.

resultante fue evaluado por medio del coeficiente de correlación cofenético (Sokal y Rolf, 1962; Steel y Torrie, 1985).

\section{Resultados}

Diversidad, estructura y formas de crecimiento. En los sitios de muestreo se registró un total de 233 taxa, incluyendo categorías infraespecíficas que engloban 55 familias, 150 géneros y 228 especies de plantas vasculares (Apéndice 1). El cuadro 1 muestra los totales para los diferentes grupos de plantas. Las dicotiledóneas comprenden el $87.7 \%$ del total de las familias, seguidas por coníferas (7.2\%) y monocotiledóneas (5.4\%). Las familias con mayor número de géneros y especies son Leguminosae, Cactaceae y Asteraceae (Figura 2) y las 11 familias más diversas aglutinan el $56 \%$ de los géneros (84) y el 58.8\% de las especies (137, Figura 2). Los nueve géneros con mayor número de especies son: Quercus (8), Acacia (7), Agave (6), Echinocereus (6), Yucca (5), Opuntia (5), Croton (5), Mammillaria (4) y Dalea (4). La mayor diversidad de especies (178) se registró en la porción húmeda del centro-sur del área del matorral submontano, en los municipios de Montemorelos y Santiago, mientras que en la porción más árida del norte, se registró un total de 112 taxa, desde el norte de Monterrey hasta el municipio de

Cuadro 1. Diversidad de familias, géneros y especies registrados en los 39 sitios de muestreo en el matorral submontano en el estado de Nuevo León.

\begin{tabular}{lcccc}
\hline & Familias & Géneros & Especies & $\begin{array}{c}\text { Categorías } \\
\text { infraespecíficas }\end{array}$ \\
\hline Pinophyta & 4 & 4 & 4 & 0 \\
\hline Magnoliophyta & & & & \\
Magnoliopsida & 48 & 139 & 207 & 1 \\
$\quad$ Liliopsida & 3 & 7 & 17 & 50 \\
\hline TOTAL & 55 & 150 & 228 & 51 \\
\hline
\end{tabular}

Cuadro 2. Principales formas de crecimiento, número de especies y sus porcentajes dentro del matorral submontano.

\begin{tabular}{lcc}
\hline Forma de crecimiento (altura) & $\begin{array}{c}\text { Número de } \\
\text { especies }\end{array}$ & $\begin{array}{c}\text { Porcentaje } \\
(\%)\end{array}$ \\
\hline Arbustos bajos $(\leq 1 \mathrm{~m})$ & 39 & 16.48 \\
Arbustos medianos $(1.1-2.5 \mathrm{~m})$ & 45 & 19.39 \\
Arbustos altos $(>2.5 \mathrm{~m})$ & 32 & 13.78 \\
Árboles & 39 & 16.77 \\
Suculentas (cactáceas) & 26 & 11.15 \\
Hierbas & 25 & 10.78 \\
Rosetófilas & 16 & 6.88 \\
Enredaderas & 9 & 3.86 \\
Parásitas & 2 & 0.88 \\
\hline TOTAL & 233 & 100.00 \\
\hline
\end{tabular}

Lampazos. Los sitios localizados en la porción centro y sur del área de estudio albergan en promedio el mayor número de especies, variando entre 34 y 89, mientras que en la parte norte los promedios por sitios son de 19 a 57 especies. Se registraron seis formas de crecimiento: árboles, arbustos, suculentas, hierbas, rosetófilas, enredaderas y parásitas. Los arbustos, árboles y suculentas presentaron el mayor número de especies (Cuadro 2). Los arbustos fueron la forma de crecimiento predominante, sumando 116 especies, de éstas, 92 son de tipo inerme y 24 de tipo espinoso (ver Apéndice 1). Las especies arbustivas del estrato medio (1.1-2.5 m) fueron las predominantes, suman 45 especies, seguidas por las del estrato bajo (39) y las de estrato alto (32). La relación entre el número de especies inermes-espinosas (i-e) en los tres estratos bajo, mediano y alto respectivamente $(b, \mathrm{~m}$ y a) del matorral fue: ib-eb $=36: 3$, im-em $=34: 12$ e ia-ea $=22: 9$. Los elementos arbustivos perennifolios (80) casi triplicaron a los elementos caducifolios (33 especies), mientras que las especies áfilas, no suculentas, sumaron sólo tres especies (ver Apéndice 1). Las 116 especies de arbustivas represen$\tan$ el $49.65 \%$ de la flora total, que sumado al $16.77 \%$ de los árboles conjuntan el $66.42 \%$ del total de los taxa. Con excepción de las rosetófilas, las restantes formas de crecimiento jugaron un papel discreto en la fisonomía del paisaje del matorral submontano.

Clasificación del matorral submontano. Se registró un total de 39 especies con valores de cobertura y densidad superiores de $0.1 \%$ y frecuencias de $5.1 \%$ o mayores, de las cuales 27 son inermes, cinco espinosas, cinco rosetófilas y dos suculentas (Cuadro 3). Diecisiete de estas especies presentaron valores de frecuencia superiores de $28.2 \%$ y son las que definieron la mayor parte de la estructura de la cobertura y densidad de esta comunidad vegetal. Los 39 sitios presentaron características edáficas heterogéneas, especialmente en textura, profundidad y contenidos de materia orgánica, 
ClasificaCión, ESTRUCTURA Y DIVERSIDAD DEL MATORRAL SUBMONTANO

Cuadro 3. Valores promedio de cobertura y densidad y valores de frecuencia de las 39 especies más importantes registradas en los 39 sitios de muestreo el matorral submontano en el estado de Nuevo León.

\begin{tabular}{|c|c|c|c|}
\hline Especie & $\begin{array}{c}\text { Cobertura } \\
(\%)\end{array}$ & $\begin{array}{l}\text { Densidad } \\
(\%)\end{array}$ & $\begin{array}{c}\text { Frecuencia } \\
(\%)\end{array}$ \\
\hline Acacia amentacea & 21.3 & 12.1 & 66.9 \\
\hline Acacia berlandieri & 3.1 & 1.1 & 30.8 \\
\hline Acacia roemeriana & 0.2 & 0.3 & 5.1 \\
\hline Agave lecheguilla & 2.6 & 3.2 & 35.8 \\
\hline Agave scabra var. scabra & 2.1 & 1.4 & 20.5 \\
\hline Aloysia macrostachya & 0.4 & 1.8 & 12.8 \\
\hline Amyris madrensis & 0.7 & 0.1 & 10.2 \\
\hline Bauhinia macranthera & 1.5 & 2.6 & 15.4 \\
\hline $\begin{array}{l}\text { Bernardia myricifolia } \\
\text { var. myrififolia }\end{array}$ & 5.2 & 2.7 & 28.2 \\
\hline Caesalpinia mexicana & 2.5 & 1.0 & 12.8 \\
\hline Colubrina greggii var. greggii & 0.1 & 1.6 & 15.4 \\
\hline Cordia boissieri & 3.2 & 2.1 & 7.7 \\
\hline Croton ciliatoglandulifer & 1.1 & 0.8 & 12.8 \\
\hline Croton suaveolens & 2.1 & 3.7 & 66.6 \\
\hline Dasylirion berlandieri & 2.1 & 3.2 & 28.2 \\
\hline Diospyros texana & 0.2 & 12.2 & 7.7 \\
\hline Eysenhardtia texana & 3.6 & 3.7 & 48.7 \\
\hline Forestiera angustifolia & 0.7 & 1.0 & 12.8 \\
\hline Fraxinus greggii var. greggii & 19.1 & 9.3 & 71.8 \\
\hline $\begin{array}{l}\text { Gochnatia hypoleuca } \\
\text { var. hypoleuca }\end{array}$ & 4.4 & 2.5 & 10.2 \\
\hline Guaiacum angustifolium & 0.1 & 1.3 & 11.7 \\
\hline Havardia pallens & 13.6 & 6.5 & 48.7 \\
\hline Helietta parvifolia & 21.4 & 13.1 & 71.8 \\
\hline Hesperaloe parviflora & 0.1 & 0.2 & 7.7 \\
\hline Jatropha dioica var. graminea & 4.1 & 4.3 & 30.8 \\
\hline Karwinskia humboldtiana & 2.2 & 4.8 & 48.7 \\
\hline Lantana macropoda & 3.2 & 5.9 & 66.6 \\
\hline Leucophyllum frutescens & 1.9 & 1.4 & 20.5 \\
\hline Lippia graveolens & 0.1 & 0.2 & 12.8 \\
\hline Neopringlea integrifolia & 14.1 & 6.7 & 66.6 \\
\hline Opuntia leptocaulis & 1.4 & 2.7 & 28.2 \\
\hline Opuntia lindheimeri & 0.5 & 1.3 & 15.4 \\
\hline Randia rhagocarpa & 3.2 & 0.2 & 20.5 \\
\hline Rhus pachyrrhachis & 1.3 & 2.7 & 28.2 \\
\hline Salvia chia & 0.6 & 1.3 & 20.5 \\
\hline Schaefferia cuneifolia & 0.4 & 2.6 & 23.1 \\
\hline Viguiera stenoloba & 0.1 & 0.8 & 7.7 \\
\hline Yucca treculeana & 0.8 & 0.1 & 7.7 \\
\hline Zanthoxylum fagara & 4.6 & 1.1 & 51.2 \\
\hline
\end{tabular}

mientras que el $\mathrm{pH}$ se mantuvo relativamente homogéneo (Cuadro 4).

Endemismo. En esta comunidad vegetal se han registrado 19 especies endémicas para el estado de Nuevo León, de las cuales 15 de ellas son de hábito herbáceo (h), tres crasicaules (cactáceas pequeñas) (cr) y un árbol (a). No hay especies
Cuadro 4. Características físicas en los 39 sitios muestreados en el matorral submontano.

\begin{tabular}{|c|c|c|c|c|c|}
\hline Sitio & Textura & $\begin{array}{l}\text { Profundidad } \\
\qquad(\mathrm{cm})\end{array}$ & $\mathrm{pH}$ & $\begin{array}{c}\text { Contenido } \\
\text { de materia } \\
\text { orgánica }(\%)\end{array}$ & $\begin{array}{c}\text { Altitud } \\
\text { (m s.n.m.) }\end{array}$ \\
\hline 1 & Arenosa & 55 & 7.3 & 3.0 & 650 \\
\hline 2 & Limosa & 25 & 7.3 & 11.9 & 600 \\
\hline 3 & Limosa & 29 & 7.3 & 7.4 & 750 \\
\hline 4 & Limosa & 25 & 7.4 & 4.9 & 550 \\
\hline 5 & Arenosa & 35 & 7.4 & 4.1 & 550 \\
\hline 6 & Arenosa & 48 & 7.4 & 4.7 & 500 \\
\hline 7 & Limosa & 45 & 7.4 & 9.1 & 520 \\
\hline 8 & Arcillosa & 45 & 7.2 & 16.0 & 500 \\
\hline 9 & Franca & 50 & 7.3 & 11.6 & 630 \\
\hline 10 & Franca & 45 & 7.1 & 7.6 & 720 \\
\hline 11 & Franco-arcillosa & 60 & 7.0 & 5.2 & 680 \\
\hline 12 & Franca & 50 & 7.1 & 3.4 & 650 \\
\hline 13 & Arenosa & 35 & 7.4 & 1.2 & 600 \\
\hline 14 & Arenosa & 20 & 7.4 & 5.4 & 500 \\
\hline 15 & Franca & 30 & 6.5 & 12.9 & 600 \\
\hline 16 & Franca & 45 & 7.4 & 6.0 & 600 \\
\hline 17 & Franca & 35 & 6.8 & 5.8 & 520 \\
\hline 18 & Franca & 45 & 7.4 & 6.0 & 560 \\
\hline 19 & Limosa & 43 & 7.0 & 3.4 & 600 \\
\hline 20 & Limosa & 30 & 7.5 & 11.1 & 500 \\
\hline 21 & Franca & 25 & 7.4 & 11.1 & 530 \\
\hline 22 & Franca & 40 & 7.2 & 12.3 & 550 \\
\hline 23 & Franco-arcillosa & 45 & 7.6 & 2.5 & 750 \\
\hline 24 & Arcillo-limosa & 38 & 7.5 & 8.6 & 570 \\
\hline 25 & Arcillo-limosa & 10 & 7.4 & 6.0 & 560 \\
\hline 26 & Franco-arcillosa & 35 & 7.3 & 8.3 & 510 \\
\hline 27 & Franco-arcillosa & 20 & 7.4 & 10.6 & 530 \\
\hline 28 & Franco-limosa & 48 & 7.7 & 1.7 & 530 \\
\hline 29 & Franca & 21 & 7.4 & 3.7 & 490 \\
\hline 30 & Franca & 32 & 7.3 & 4.2 & 420 \\
\hline 31 & Franco-limosa & 38 & 7.3 & 5.7 & 520 \\
\hline 32 & Franco-limosa & 29 & 7.3 & 8.5 & 610 \\
\hline 33 & Franco-arcillosa & 33 & 7.2 & 7.7 & 630 \\
\hline 34 & Franca & 46 & 7.3 & 1.7 & 485 \\
\hline 35 & Franco-limosa & 27 & 7.4 & 8.9 & 530 \\
\hline 36 & Franco-limosa & 41 & 7.3 & 8.9 & 650 \\
\hline 37 & Franco-limosa & 52 & 7.2 & 7.2 & 575 \\
\hline 38 & Arcillo-limosa & 38 & 7.1 & 7.2 & 495 \\
\hline 39 & Franco-limosa & 44 & 7.3 & 7.3 & 560 \\
\hline
\end{tabular}

arbustivas endémicas en el matorral submontano. Las especies endémicas son: Agave albopilosa I. Cabral, Villarreal \& A.E.Estrada, Asplenium ultimum A.R.Sm (h), Allium traubii T.M.Howard (h), Ariocarpus scaphirostris Boed. (cr), Cynanchum maccartii Shinners var. latifolium B.L.Turner ex Henrickson (h), Desmanthus pringlei (Britton \& Rose) F.J.Herm. (h), Epithelantha micromeris F.A.C.Weber var. unguispina (Boed.) N.P.Taylor (cr), Flyriella leonensis (B.L.Rob.) R.M.King \& H.Rob. (h), Linum lasiocarpum 


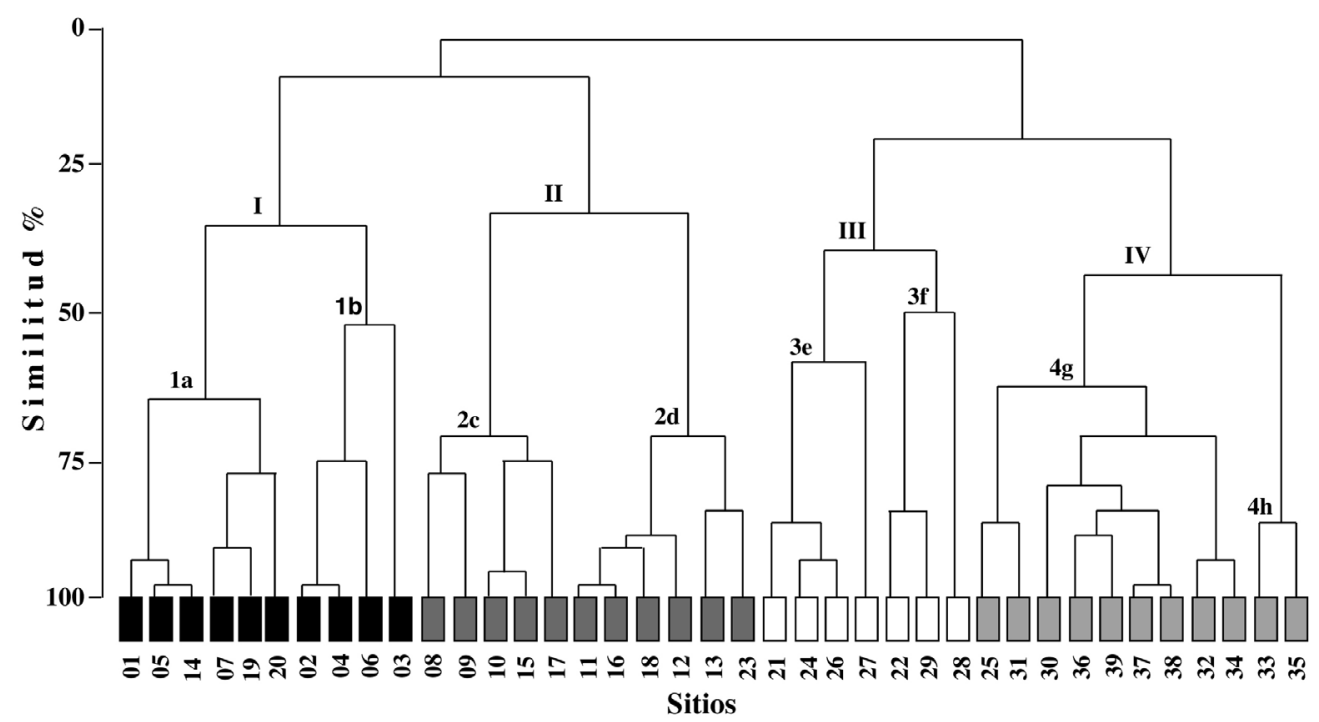

Figura 3. Dendrograma mostrando el agrupamiento de los 39 sitios dentro del matorral submontano en el estado de Nuevo León, México.

Rose (h), Lithospermum nelsonii Greenm. (h), Mammillaria linaresensis R.Wolf \& F.Wolf (cr), Mirandea huastecensis T.F.Daniel (h), Myrospermum sousanum A.Delgado \& M.C.Johnston (a), Perymenium hintoniorum B.L.Turner var. hintoniorum (h), Phaseolus novoleonensis Debouck (h), Romanschulzia meyeri Rollins (h), Seymeria deflexa Eastw. (h), Thelesperma subaequale S.F.Blake (h) y Vigethia mexicana (S.Watson) W.A.Weber (h) (Villarreal-Quintanilla y Estrada-Castillón, 2008). Con excepción de Myrospermum sousanum, el resto de las especies no aparecieron en los sitios de muestreo realizados.

Agrupación. El dendrograma resultante mostró una correlación de $\mathrm{r}=0.86$ y un coeficiente de correlación cofenético de $r=0.84$. A un nivel de similitud de $37 \%$ se reconocen cuatro grupos y ocho diferentes asociaciones de plantas en el matorral submontano (Figura 3). La agrupación de sitios de los grupos I y II se caracterizó por los altos valores de cobertura y densidad de las especies dominantes asociadas, Acacia amentacea, Diospyros texana, Gochnatia hypoleuca var. hypoleuca, Havardia pallens, Helietta parvifolia, Fraxinus greggii var. greggii, Neopringlea integrifolia, Randia rhagocarpa y Zanthoxylum fagara. Estas especies constituyen matorrales medianos (1-2.5 m altura) y altos $(>2.5$ $\mathrm{m})$, localizados en la parte central y sur del área de estudio, mientras que los grupos III y IV están agrupados por los valores de cobertura y densidad de la asociación de las especies Acacia amentacea, A. berlandieri, Agave lecheguilla, Amyris madrensis, Bernardia myricifolia var. myricifolia, Cordia boissieri, Eysenhardtia texana, Helietta parvifolia, Jatropha dioica var. graminea, Leucophyllum frutescens, Neopringlea integrifolia y Schaefferia cuneifolia, mismas que conforman matorrales de tipo bajo y mediano, entre 1 $\mathrm{y} \leq 2.5 \mathrm{~m}$ de altura, distribuidos principalmente en la parte centro-norte y norte del área de estudio.
Grupo I. Incluye diez sitios, puede ser dividido en dos subgrupos. El subgrupo Ia correspondió a una asociación de arbustos altos, dominado por especies inermes con altos valores de cobertura y densidad, entre las que destacan Acacia amentacea, Fraxinus greggii var. greggii, Gochnatia hypoleuca var. hypoleuca, Havardia pallens, Helietta parvifolia y Neopringlea integrifolia, acompañadas por un estrato medio de arbustos inermes y con menor cobertura y dominado por Amyris madrensis, Bauhinia macranthera, Bernardia myricifolia var. myricifolia, Croton suaveolens, Forestiera angustifolia, Karwinskia humboldtiana y Lantana macropoda. El subgrupo $I b$ incluyó arbustos inermes de altura media (Colubrina greggii var. greggii, Fraxinus greggii var. greggii, Gochnatia hypoleuca var. hypoleuca y Helietta parvifolia), inermes de altura baja (Bernardia myricifolia var. myricifolia, Croton suaveolens y Lantana macropoda) y arbustos espinosos medianos (Acacia amentacea) y altos (Havardia pallens). Estas asociaciones se distribuyen principalmente en las partes más bajas de las montañas en la porción sur y en las faldas de las montañas aisladas en la región central del área de estudio, desde el municipio de Santiago hasta el municipio de Linares. Los suelos en estos sitios presentan principalmente textura arenosa y limosa, con profundidades entre los 25 y $48 \mathrm{~cm}, \mathrm{pH}$ de 7 a 7.5 y contenidos de materia orgánica entre 3 y $11.9 \%$, en altitudes entre los 500 y $750 \mathrm{~m}$.

Grupo II. Aglutinó 11 sitios localizados en la porción más húmeda del sur, en las partes medias de las montañas de los municipios de Santiago, Allende y Montemorelos y un cuadrante de la porción norte (municipio Villaldama). Se reconocieron dos subgrupos con base en la composición de especies. El subgrupo IIc fue una asociación de arbustos espinosos medianos con Acacia amentacea y Randia rhagocarpa así como arbustos inermes altos como Caesalpinia 
mexicana, Diospyros texana y Fraxinus greggii var. greggii. En ocasiones este matorral conforma intrincadas asociaciones con las comunidades de árboles presentes de la cota altitudinal superior en áreas húmedas o en cañones amplios, donde se encuentran especies de los géneros Carya, Cephalanthus, Juglans, Platanus, Salix, Taxodium, Ulmus y Ungnadia. En este subgrupo se registró la mayor diversidad de especies arbustivas (178). El subgrupo IId incluyó seis sitios y al igual que el subgrupo anterior, está constituido por arbustos inermes y espinosos medianos y altos. Las principales especies de acuerdo con sus valores de importancia son Havardia pallens, Neopringlea integrifolia y Zanthoxylum fagara, acompañadas por Croton suaveolens, Eysenhardtia texana, Karwinskia humboldtiana y Lantana macropoda, aunque con valores de cobertura menores. Esta asociación se localiza principalmente en las faldas bajas de montañas en las regiones del centro del área de estudio (partes de Santiago, municipios de Monterrey, San Pedro Garza García, Santa Catarina, Guadalupe, Juárez e Hidalgo). Los suelos de estas porciones son principalmente de textura arcillosa, franco-arcillosa y franca, con profundidades entre los $30 \mathrm{y}$ $60 \mathrm{~cm}, \mathrm{pH}$ entre 6.5 y 7.4 y contenidos de materia orgánica entre 1.2 y $16 \%$, en altitudes entre los 500 y $750 \mathrm{~m}$. La inclusión del sitio 23 dentro de este subgrupo, aún y cuando no pertenece al área húmeda de la región se debe a los altos valores de cobertura de $H$. pallens (valores mucho mayores que el resto de sitios de la porción norte).

Grupo III. Incluyó siete sitios de la porción central y norte, desde Monterrey, Sabinas Hidalgo, Salinas Victoria, Mina hasta partes del municipio Bustamante y se reconocieron dos subgrupos. En el subgrupo IIIe, la mayoría de las especies arbustivas presentes en estos sitios fueron rosetófilas (Agave lecheguilla) y suculentas (Opuntia engelmannii) así como arbustos inermes altos (hasta $3 \mathrm{~m}$ de altura) (Helietta parvifolia) y bajos (Karwinskia humboldtiana), mientras que elementos suculentos (Opuntia leptocaulis) e inermes bajos (Jatropha dioica var. graminea, Leucophyllum frutescens y Schaefferia cuneifolia) constituyeron el subgrupo IIIf. El suelo en estos sitios presenta textura franca, francoarcillosa y franco-limosa, son relativamente someros, con profundidades entre los 21 y $40 \mathrm{~cm}, \mathrm{pH}$ relativamente homogéneo, entre 7.2 y 7.7 y contenidos de materia orgánica entre 1.7 y $12.3 \%$, estos sitios se localizan en altitudes entre los 490 y $570 \mathrm{~m}$, principalmente en lomeríos bajos.

Grupo IV. Agrupó 11 sitios, todos ellos localizados en la porción del norte, en los municipios de Bustamante, Villaldama y Lampazos, al igual que el grupo anterior, el paisaje es dominado por la cobertura de arbustos inermes de porte medio, así como por especies rosetófilas. Las especies dominantes en este grupo fueron Acacia amentacea, A. berlandieri, Agave lecheguilla, A. scabra var. scabra, Bernardia myricifolia var. myricifolia, Eysenhardtia texana, Forestiera angustifolia, Helietta parvifolia, Jatropha dioica var. graminea, Leucophyllum frutescens, Rhus pachyrrha-
Cuadro 5. Áreas naturales dedicadas a la conservación de recursos naturales en el estado de Nuevo León y hectáreas (ha) de matorral submontano que comprenden.

\begin{tabular}{lc}
\hline Areas Naturales & $\begin{array}{c}\text { Matorral } \\
\text { submontano (ha) }\end{array}$ \\
\hline Parque Nacional Cumbres de Monterrey & 49,839 \\
Monumento Nacional Cerro de La Silla & 3,255 \\
Zona sujeta a conservación ecológica Cerro El Topo & 1,093 \\
Zona sujeta a conservación ecológica Cerro La Mota & 1,769 \\
Parque Urbano Río Santa Catarina & 4 \\
$\begin{array}{l}\text { Zona sujeta a conservación ecológica Sierra Cerro } \\
\text { de La Silla }\end{array}$ & 7,481 \\
$\begin{array}{l}\text { Zona sujeta a conservación ecológica Sierra } \\
\quad \text { El Fraile y San Miguel }\end{array}$ & 16,430 \\
Zona sujeta a conservación ecológica Sierra Las Mitras & 1,388 \\
Zona sujeta a conservación ecológica Sierra Picachos & 62,290 \\
Zonas de restauración ecológica del lobo mexicano & 72.4 \\
$\quad$ Corona del Rosal & \\
Zonas de restauración ecológica del lobo mexicano & 727 \\
$\quad$ Potrero de Zamora & \\
Zonas de restauración ecológica del lobo mexicano & 416 \\
$\quad$ San Joaquín de Soto & \\
\hline
\end{tabular}

chis y Schaefferia cuneifolia, la mayoría de éstas son dominantes en el subgrupo $I V g$, y se localizan en las partes medias de la Sierra de Bustamante y Sierra de Lampazos; mientras que en el subgrupo $I V h$, distribuido en las partes bajas de la Sierra de Lampazos, predominaron las especies rosetófilas así como arbustos de porte bajo y medio, algunos de ellos ausentes en los grupos y subgrupos anteriores como Agave scabra var. scabra, Aloysia macrostachya, Bauhinia macranthera, Brahea berlandieri, Colubrina greggii var. greggii, Dasylirion texanum, Guaiacum angustifolium, Hesperaloe parviflora, Lippia graveolens, Parthenium incanum, Salvia chia, Viguiera stenoloba y Yucca treculeana. Los suelos en este subgrupo son de textura franca, francolimosa y franco-arcillosa, con 10 a $52 \mathrm{~cm}$ de profundidad, $\mathrm{pH}$ de 7.1 a 7.4 y contenidos de materia orgánica entre 1.7 y $8.9 \%$, en altitudes que van de $\operatorname{los} 420$ a los $630 \mathrm{~m}$.

Situación actual del matorral submontano en Nuevo León. En Nuevo León existen 23 áreas naturales estatales protegidas, un monumento nacional (Cerro La Silla), un parque urbano (Parque lineal, Río Santa Catarina) y tres Área Naturales Protegidas (ANP) comunitarias (Zonas de restauración ecológica del lobo mexicano) (Secretaría de Desarrollo Urbano y Obras Públicas, 2000) mismas que comprenden una superficie total de 150,931 ha $\left(1,509.3 \mathrm{~km}^{2}\right)$. El matorral submontano se localiza en parte o totalmente en 12 de estas áreas naturales para la conservación ecológica (Figura 4, Cuadro 5). En conjunto, suman cerca de 144,764 ha, de las cuales 81,261 ha se localizan en el centro del estado, próximos al área metropolitana y hasta los municipios de Allende y Montemorelos. Una pequeña porción se registra 


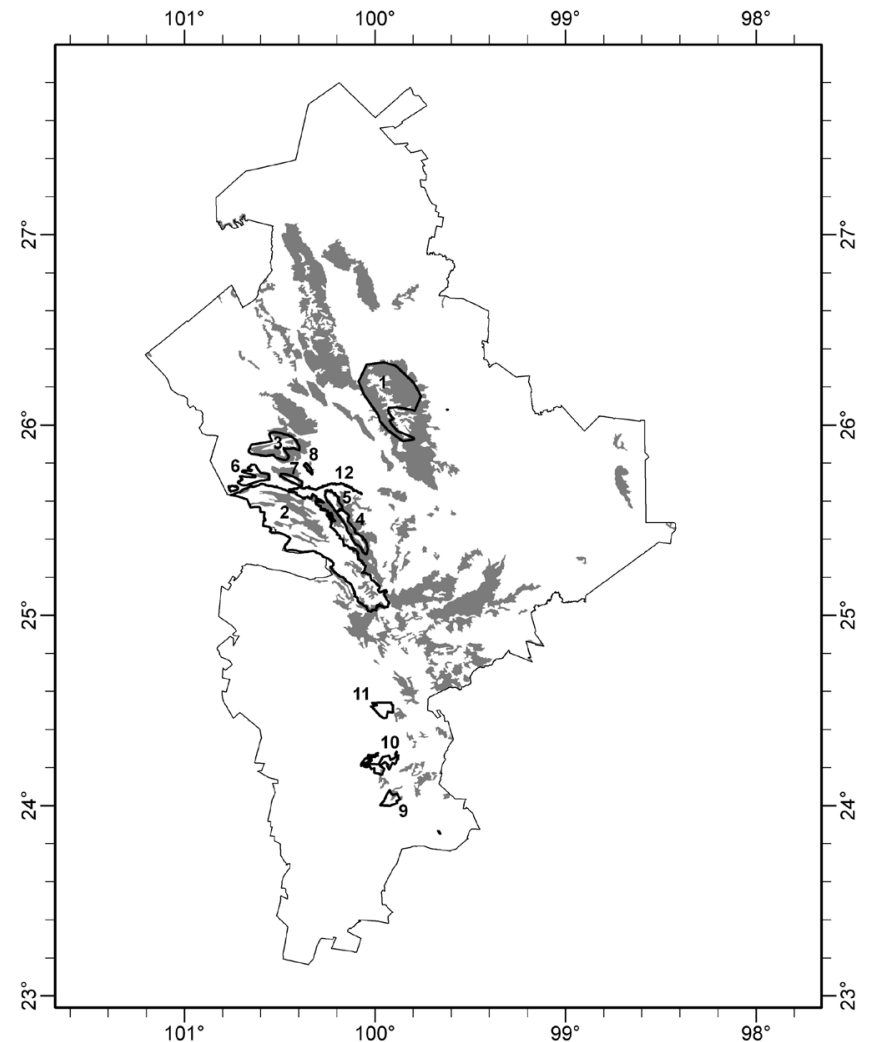

Figura 4. Áreas naturales en el estado de Nuevo León donde se registra la presencia de matorral submontano: 1) Sierra Picachos, 2) Parque Nacional Cumbres de Monterrey, 3) Sierra El Fraile $y$ San Miguel, 4) Sierra Cerro La Silla, 5) Cerro La Silla, 6) Cerro La Mota, 7) Sierra Las Mitras, 8) Cerro El Topo, 9, 10 y 11) Áreas de restauración ecológica del lobo mexicano, 12) Parque lineal Río Santa Catarina.

en la región sur y el resto se localiza en la región centronorte $(62,290$ ha).

\section{Discusión}

Diversidad arbustiva e importancia de las especies del matorral submontano. Las especies más importantes y dominantes en el matorral submontano son Acacia amentacea, $A$. berlandieri, Agave lecheguilla, Bernardia myricifolia var. myricifolia, Croton suaveolens, Dasylirion berlandieri, Eysenhardtia texana, Fraxinus greggii var. greggii, Havardia pallens, Helietta parvifolia, Jatropha dioica var. graminea, Karwinskia humboldtiana, Lantana macropoda, Neopringlea integrifolia, Rhus pachyrrhachis y Zanthoxylum fagara. Estas especies definen, en general, todas las asociaciones vegetales del matorral submontano en el estado de Nuevo León. A pesar de su alta diversidad en comparación con otras familias, las especies de Asteraceae y Cactaceae presentan bajos valores de cobertura, densidad y frecuencia en las diferentes asociaciones que componen este matorral comparados con miembros de Leguminosae, Oleaceae, Ru- taceae y Boraginaceae. Los tres taxa más frecuentes con los más altos valores de cobertura y densidad y que definen en el mayor de los casos el matorral submontano son Helietta parvifolia, Fraxinus greggii var. greggii y Neopringlea integrifolia. Estas especies generalmente predominan en las pendientes pronunciadas o someras de las cadenas montañosas y aún en sierras aisladas, rara vez se mezclan con las comunidades de suelos planos o de planicie (espacialmente con matorral espinoso tamaulipeco o matorral micrófilo de Larrea y Flourensia en el norte del estado) o cuando lo hacen, no son los taxa dominantes. El elemento característico del matorral submontano es Helietta parvifolia, especie que se encuentra a manera de un cinturón de vegetación entre los 450 y $700 \mathrm{~m}$ de altitud a lo largo de su distribución nortesur. Las asociaciones vegetales donde predomina Helietta parvifolia son conspicuas por su fisonomía de matorral alto y de color verde ceniciento, brilloso, fácilmente distinguible del verde de las otras asociaciones vegetales adyacentes.

En Nuevo León, el matorral submontano da paso a los encinares en el nivel altitudinal superior. En el norte del estado, ocasionalmente da paso al bosque escuamifolio ( $\mathrm{Ju}$ niperus pinchotii) o de coníferas (Pinus cembroides) en las serranías bajas con altiplano. En ocasiones se constituyen ecotonos de estas comunidades, donde las especies de encino, coníferas y de matorral aumentan la diversidad en estas áreas, de ahí que el género Quercus (ocho especies) muestre una diversidad superior aún a Acacia (siete especies), el arbusto más diverso en el matorral submontano.

Afinidades ecológicas y número de taxa. La relación cuantitativa de especies inermes y espinosas en los diferentes estratos de vegetación dan un claro indicativo del predominio de los elementos inermes sobre los espinosos en los tres estratos, especialmente en el bajo y en el alto. Las especies y la vegetación en conjunto, localizadas en la porción media y sur de esta comunidad vegetal promedian las mayores alturas (3 a $5.5 \mathrm{~m}$ ) y porcentajes de cobertura entre 37 y $78 \%$. Los suelos de estos sitios presentan textura generalmente arenosa y arenoso-arcillosa. En la porción norte, la altura promedio de las plantas es de 1.5 a $3 \mathrm{~m}$ y los suelos son de textura principalmente areno-arcillosa y limoso-arcillosa. Aunado al tipo de suelo, existen factores que modifican drásticamente la conformación de la vegetación del matorral submontano en forma similar a como sucede en otros tipos de matorral xerófilo en el sur de México, donde destacan la topografía accidentada, los microambientes, la profundidad de suelo y la pendiente (Santibáñez-Andrade et al., 2009).

Las asociaciones de matorral submontano en el sur y centro del estado alcanzan mayores alturas, densidades y coberturas que en la región norte. Dos factores pueden ser la causa de este efecto, la precipitación y el suelo. La precipitación (980 a 1,020 mm anuales) en la región centro-sur, duplica a la que recibe la región norte, mientras que los suelos de la región centro-sur son, en general, más profundos y 
relativamente con mayor contenido de materia orgánica que los del norte.

Asociaciones vegetales como las que conforma Helietta parvifolia en el norte del área de estudio han sido descritas para el norte el estado de Nuevo León (Briones y VillarrealQuintanilla, 2001) y en, Guanajuato, Querétaro y San Luís Potosí (Rzedowski 1966, 1978). En los cañones más húmedos y en áreas riparias de la región central del área de estudio, el matorral submontano se mezcla con especies arbóreas que no son encinos ni coníferas, destacan especies de Carya, Cephalanthus, Juglans, Platanus, Salix, Taxodium, Ungnadia y Ulmus, asociaciones vegetales afines en composición y fisonomía a las reportadas por Marroquín (1968) para el centro del estado. En las áreas húmedas, a lo largo de la región occidental en México, específicamente Baja California, la Península de Yucatán y el Valle de TehuacánCuicatlán se desarrollan comunidades de plantas conocidas como bosque tropical seco (Bullock et al., 1995) donde dominan las especies tropicales que alcanzan alturas entre 8 y $9 \mathrm{~m}$ de altura (Trejo-Vázquez, 1999). Características similares se presentan en el matorral submontano: especies de afinidad tropical asociadas con suculentas, pero dominado por especies arbustivas medianas o altas $(2.5-4.5 \mathrm{~m})$. De acuerdo con Cain (1950), las plantas adaptadas a un particular régimen climático frecuentemente ostentan similares formas biológicas. En este caso, existen ciertas afinidades entre el bosque tropical seco y el matorral submontano, pues ambas comunidades presentan un período de sequía y se distribuyen principalmente en climas de tipo cálidosubhúmedo. Además en ambos se encuentran pendientes moderadas o pronunciadas y también diversos tipos de suelo, factor que podría explicar, como argumentan Trejo-Vázquez (1999) y Gallardo-Cruz et al. (2005) la diversidad de especies en ambas comunidades vegetales. Dos matorrales afines al submontano son los denominados matorral tropical del Cabo y selva baja del Cabo (pie de monte de las montañas de la Región del Cabo) (González-Abraham et al., 2010), ambos localizados en la región de El Cabo, Baja California. En ellos los elementos predominantes del primero son matorrales bajos, donde destacan algunas especies de los géneros Colubrina, Esenbeckia, Fouquieria, Havardia, Karwinskia, Parkinsonia y Prosopis, todos presentes en el matorral submontano. El segundo, localizado entre los 500 y $1,000 \mathrm{~m}$ de altitud se caracteriza por especies caducifolias (al menos nueve meses del año) de los géneros Disopyros, Erythrina, Gochnatia, Jatropha, Lysiloma, Quercus, Senna y Zanthoxylum, igualmente presentes (excepto Lysiloma) en el matorral submontano. Las comunidades de matorral submontano presentes en Nuevo León, Coahuila, San Luís Potosí y Zacatecas son relativamente homogéneas en estructura, predominando las especies arbustivas, inermes de porte mediano (hasta de $2.5 \mathrm{~m}$ ), a diferencia de algunas comunidades vegetales como el bosque tropical caducifolio del sur de México, donde los elementos predominantes son árboles o cactáceas columnares (Durán et al., 2006).

Aunque la mayoría de las especies registradas tienen una amplia distribución dentro del matorral submontano, varias de ellas se encuentran restringidas a climas y suelos específicos. Por ejemplo, algunos miembros de Agavaceae, Agave bracteosa, A. victoriae-reginae y A. albopilosa (litosoles), Brahea berlandieri (suelos pedregosos en las partes altas de serranías áridas), Dasylirion berlandieri (pendientes), Hesperaloe funifera var. funifera (suelos pedregosos) y Yucca filifera (suelos profundos).

El matorral submontano en Nuevo León presenta 55 familias, 150 géneros y 228 especies de plantas vasculares, que comparado con las 24,424 especies, 2,804 géneros y 304 familias registradas para México (Villaseñor, 2004), representan el $1 \%, 5.34 \%$ y $1.81 \%$ de su flora, respectivamente. Además, sobrepasa por mucho al matorral de tipo costero de Yucatán, donde se registran 71 especies, 66 géneros y 36 familias de plantas vasculares (Torres et al., 2010), con valores similares en cuanto a número de especies para algunos sitios del bosque tropical seco estacional en el centro y sur de México (Trejo y Dirzo, 2002). También es afín con respecto al número de familias y especies registradas para el bosque tropical caducifolio de la región de Nizanda, en el Itsmo de Tehuantepec (Pérez-García y Meave, 2004) o a los matorrales crasicaules y espinosos y manchones de bosque tropical caducifolio de la porción central de Querétaro (Cabrera-Luna y Gómez-Sánchez, 2005), pero por debajo de los registros de familias (68), géneros (206) y especies (324) para la región desértica de San Felipe, Baja California (Delgadillo-Rodríguez y Macías-Rodríguez, 2002) y para las selvas bajas de corrientes de lava en Veracruz, con 104 familias, 394 géneros y 666 especies (Castillo-Campos et al., 2007).

El matorral submontano su endemismo y conservación en Nuevo León. A pesar de ser uno de los tipos de vegetación donde se llevan a cabo múltiples actividades económicas como desarrollo de asentamientos humanos e industria, el matorral submontano ostenta poblaciones bien conservadas en las laderas y pendientes de montañas en el norte, centro y sur del estado de Nuevo León, que representan fielmente su fisonomía de matorral mediano, alto o bajo, especialmente de tipo subinerme. A diferencia de otros matorrales del sur de México, donde la principal afectación es causada por invasión de exóticas, contaminación, saqueo de especies y fuegos (Castillo-Argüero et al., 2004), el matorral submontano se ve afectado principalmente por la tala total para desarrollos urbanos.

Sin lugar a dudas, los sitios de matorral submontano con mayor deterioro se localizan en las zonas adyacentes al área metropolitana. De acuerdo con los mapas de uso y vegetación Serie II (INEGI, 2008) y Serie III (INEGI, 2009), la superficie ocupada por el área metropolitana en el año 2000 era de $295 \mathrm{~km}^{2}$, mientras que en 2005 era de $388 \mathrm{~km}^{2}$ 
y en el año 2007 de $409 \mathrm{~km}^{2}$. En siete años, se eliminaron 11,400 ha de este tipo de vegetación en el área metropolitana, especialmente para desarrollo urbano. Las áreas más afectadas por esta actividad se localizan en los alrededores de la Sierra El Fraile y San Miguel, Cerro El Topo, Cerro La Silla, Sierra La Silla y el Parque Nacional Cumbres de Monterrey. La Sierra Picachos en el centro-norte del estado alberga matorral submontano en condiciones relativamente óptimas, pues la principal actividad económica es la extracción de leña practicada por los pobladores de la región, así como ramoneo por ganado caprino (obs. pers.), sin presentar actividades de desmonte de grandes áreas para desarrollos urbanos como sucede en el centro del estado.

La mayoría de las especies endémicas para el estado de Nuevo León presentes en el matorral submontano poseen distribución restringida o, en ocasiones, se registran en las comunidades vegetales adyacentes. Las especies en mayor riesgo son las cactáceas, al encontrarse en las áreas de matorral submontano con mayor impacto directo e indirecto por parte del hombre y no se localizan dentro de ninguna área natural protegida del estado. Varias especies de cactáceas que no son endémicas del matorral submontano, pero están presentes en él y se encuentran bajo algún estatus de protección en México (SEMARNAT, 2002) son Coryphantha sulcata var. nickelziae, Echinocereus poselgeri, Ferocactus haematacanthus, Mammillaria plumosa y Thelocactus bicolor. Agave victoriae-reginae es una especie endémica de México (NOM-059-ECOL-2001) catalogada como en peligro de extinción presente en los taludes y acantilados del Cañón La Huasteca en los municipios de Santa Catarina y Rayones; sitios de fácil acceso y la escasa protección de estas poblaciones silvestres ha contribuido a su extracción ilegal y destrucción de parte de su hábitat. Agave albopilo$s a$ tiene una distribución más restringida que $A$. victoriaereginae, se localiza únicamente en el municipio de Santa Catarina, en los acantilados más altos, también en el Cañón de La Huasteca, aún no se conoce mucho de la biología y por su reciente descubrimiento (2010) no está registrado su estatus de riesgo en el país (Cabral-Cordero et al., 2007).

Una leguminosa presente en el matorral submontano con distribución restringida es Myrospermum sousanum, únicamente registrada en dos montañas en el estado de Nuevo León (Estrada-Castillón et al., 2005), una de ellas localizada en el municipio de Villaldama y la otra en el municipio de Bustamante, adyacente al atractivo turístico regional, las Grutas de Bustamante. En las Grutas de Bustamante contabilizamos menos de 200 individuos (es la población de mayor tamaño), pero en los años 2009-2010 se realizaron trabajos de mejoramiento de caminos de acceso a las grutas y al menos diez árboles de entre 6 y $8 \mathrm{~m}$ de altura fueron tumbados (obs. pers.), reduciendo aun más sus poblaciones.

Dentro de las ANP's y otras áreas de protección a los recursos naturales en el estado de Nuevo León donde se encuentra la presencia de matorral submontano se registraron los tres estratos de vegetación que componen su fisonomía general. Esto se interpreta como una condición óptima de su fisonomía donde se albergan las asociaciones de especies características aquí registradas. Sin embargo, en áreas fuera de la protección gubernamental o propiedad privada, como sucede en áreas de los alrededores de Montemorelos, Allende, Santiago, Hualahuises y Linares, las poblaciones de matorral submontano alto, donde dominan en densidad individuos de Dispyros, Fraxinus y Helietta, están siendo fuertemente impactadas por efecto de la tala selectiva de estas especies para utilizarlas como postes para cerca, leña y, especialmente, para la industria artesanal (yoyos, trompos, baleros) en el municipio de Hualahuises. Sería deseable que las autoridades gubernamentales atendieran este problema, considerando que las poblaciones de matorral submontano alto son cada vez más escasas en el centro y centro-sur del estado, que es el área con mayor diversidad de especies de arbustos y es albergue de la mayor parte de las especies endémicas dentro de esta comunidad vegetal.

En el noreste de México y en colindancia con la frontera con los Estados Unidos, en áreas donde predomina el matorral submontano, se registran varios cientos de UMA's encaminadas al aprovechamiento cinegético de fauna mayor. Desde el punto de vista de conservación, se están manejando y protegiendo estas asociaciones vegetales mediante su uso como fuente de alimento de fauna silvestre, reproducción de venado cola blanca, jabalí y guajolote. Estas UMA's son en la actualidad uno de los negocios más redituables económicamente, sobrepasando en ganancias las prácticas antiguas de manejo de ganado (SEMARNAT, 2007).

\section{Agradecimientos}

Este trabajo fue patrocinado por el Consejo Nacional de Ciencia y Tecnología (CONACYT 34033-V) y parcialmente por el Programa de Apoyo a la Investigación Científica y Tecnológica (PAICYT) de la Universidad Autónoma de Nuevo León. A Carmen Yen Méndez, Guadalupe Cavazos García y Margarita Barba Platas por su apoyo y asistencia en el trabajo de campo. A dos revisores anónimos por sus comentarios con los cuales se mejoró sustancialmente el contenido del escrito.

\section{Literatura citada}

Alanís-Flores G.J. 2004. Florística de Nuevo León. En: Luna I., Morrone J.J. y Espinoza D. Eds. Biodiversidad de la Sierra Madre Oriental, pp. 243-258, Comisión Nacional para el Conocimiento y Uso de la Biodiversidad/Universidad Nacional Autónoma de México, México, D.F.

Bonham C.D. 1980. Measurements for Terrestrial Vegetation. Wiley \& Sons, NuevaYork.

Bullock S.H., Mooney H.A. y Medina E. Eds. 1995. Seasonally Dry Tropical Forest.Cambridge University Press, Cambridge.

Briones O. y Villarreal-Q. J.A. 2001. Vegetación y flora de un eco- 
tono entre las provincias del Altiplano y de la Planicie Costera del Noreste de México. Acta Botanica Mexicana 55:39-67.

Briones-Villarreal O.L. 1991. Sobre la flora, vegetación y fitogeografía de la Sierra de San Carlos, Tamaulipas. Acta Botanica Mexicana 16:15-43.

Cabral-Cordero I., Villarreal-Quintanilla J.A. y Estrada-Castillón A.E. 2007. Agave albopilosa (Agavaceae, Subgénero Littaea, grupo Striatae), una nueva especie de la Sierra Madre Oriental en el noreste de México. Acta Botanica Mexicana 80:51-57.

Cain S.A. 1950. Life-forms and phytoclimate. Botanical Review 16:1-32.

Cabrera-Luna J.A. y Gómez-Sánchez M. 2005. Análisis florístico de La Cañada, Querétaro, México. Boletín de la Sociedad Botánica de México 77:35-50.

Castillo-Argüero S., Montes-Cartas G., Romero-Romero M.A., Martínez-Orea Y., Guadarrama-Chávez P., Sánchez-Gallén I. y Núñez-Castillo O. 2004. Dinámica y conservación de la flora del matorral xerófilo de la Reserva Ecológica del Pedregal de San Ángel (D.F., México). Boletín de la Sociedad Botánica de México 74:51-75.

Castillo-Campos G., Dávila-Aranda P. y Zavala-Hurtado J.A. 2007. La selva baja caducifolia en una corriente de lava volcánica en el centro de Veracruz: Lista florística de la flora vascular. Boletín de la Sociedad Botánica de México 80:77-104.

Challenger A. 1998. Utilización y Conservación de los Ecosistemas Terrestres de México. Pasado, Presente y Futuro. Comisión Nacional para el Conocimiento y Uso de la Biodiversidad/ Universidad Nacional Autónoma de México/Agrupación Sierra Madre S. C., México, D.F.

CONABIO [Comisión Nacional para el Conocimiento y Uso de la Biodiversidad]. 2010. Portal de Geoinformación. en: <www. conabio.gob.mx/informacion/gis/> (consultado 5 noviembre 2011).

Delgadillo-Rodríguez J. y Macías-Rodríguez M.A. 2002. Componente florístico del Desierto de San Felipe, Baja California, México. Boletín de la Sociedad Botánica de México 70:45-65.

Digby P.G.N. y Kempton R.A. 1991. Multivariate Analysis of Ecological Communities. Chapman \& Hall, Nueva York.

Durán E., Meave J.A., Lot E.J. y Segura G. 2006. Structure and tree diversity patterns at the landscape level in a Mexican tropical deciduous forest. Boletín de la Sociedad Botánica de México 79:43-60.

Estrada-Castillón E., Villarreal-Quintanilla J.A. y Jurado E. 2005. Leguminosas del norte del estado de Nuevo León, México. Acta Botanica Mexicana 73:1-18.

Gallardo-Cruz J., Meave J.A. y Pérez-García E.A. 2005. Estructura, composición y diversidad de la selva baja caducifolia del Cerro Verde, Nizanda (Oaxaca), México. Boletín de la Sociedad Botánica de México 76:19-35.

García E. 1973. Modificaciones al Sistema de Clasificación Climática de Koëpen. Universidad Nacional Autónoma de México, Instituto de Geografía, México, D.F.

Gauch H.G.1982. Multivariate Analysis in Community Ecology. Cambridge University Press, Cambridge.

Giménez-de Azcárate J. y González-Costilla O. 2011. Pisos de vegetación de la Sierra de Catorce y territorios circundantes (San Luís Potosí, México). Acta Botanica Mexicana 94:91-123.

González-Abraham C.E., Garcillán P.P., Excurra E. y el Grupo de Trabajo de Ecorregiones. 2010. Ecorregiones de la península de Baja California: una síntesis. Boletín de la Sociedad Botánica de México 87:69-82.

González-Costilla O., Jiménez-de Azcárate J., García-Pérez J. y Aguirre-Rivera J.R. 2007. Flórula vascular de la Sierra de Catorce y territorios adyacentes, San Luís Potosí, México. Acta Botanica Mexicana 78:1-38.

González-Medrano F. 1972. La vegetación del nordeste de Tamaulipas. Anales del Instituto de Biología. Universidad Nacional Autónoma de México, Serie Botánica 43:11-50.

Gutiérrez-Lobatos J.L. 1970. El matorral submontano en los alrededores de Monterrey, Nuevo León. Tesis de Licenciatura, Facultad de Ciencias Biológicas, Universidad Autónoma de Nuevo León, San Nicolás de los Garza. 74 Pp.

Herrera A.H. 1993. Una Mirada al Campo de la Clasificación Numérica. Instituto de Oceanología, Academia de Ciencias de Cuba, La Habana.

INEGI. 1986a. Carta de climas, Monterrey, escala 1: 1,000000. Secretaría de Programación y Presupuesto, México, D.F.

INEGI. 1986b. Cartas de uso de suelo y vegetación escala 1: 150,000. G14A44, G14A45, G14A54, G14A55, G14A64, G14A65, G14A75, G14A84, G14A85, C14C46, G14C56, G14C57, G14C58, G14C77. Secretaría de Programación y Presupuesto, México, D.F.

INEGI. 1986c. Síntesis geográfica de Nuevo León. Secretaría de Programación y Presupuesto, México, D.F.

INEGI. 2008. Carta digital, uso de suelo y vegetación, escala 1: 250,000. Serie II. Instituto Nacional de Estadística, Geografía e Informática, México, D.F.

INEGI. 2009. Carta digital, uso de suelo y vegetación, escala 1: 250,000. Serie III. Instituto Nacional de Estadística, Geografía e Informática, México, D.F.

Kovach Computing Services. 1998. MultiVariate Statistical Package. Anglesey.

Manly B.F.J. 1992. Multivariate Statistical Methods: A Primer. Chapman and Hall, Londres.

Marroquín J.S., Borja G., Velázquez R. y de la Cruz J.A. 1964. Estudio ecológico dasonómico de las zonas áridas del norte de México. Instituto. Nacional de Investigaciones. Forestales. Publ. Esp. 2. México, D.F.

Marroquín J.S. 1968. Datos botánicos de los cañones orientales de la Sierra de Anáhuac al sur de Monterrey. No 14 de los Cuadernos del Instituto de Investigaciones Científicas de la Universidad Autónoma de Nuevo León, San Nicolás de los Garza.

Melgoza-Castillo A. 1977. Estudio florístico ecológico de comunidades secundarias de matorral submontano en Santiago, Nuevo León, México. Tesis de Licenciatura, Facultad de Ciencias Biológicas, Universidad Autónoma de Nuevo León, San Nicolás de los Garza, 75 pp.

Miranda F. y Hernández-X.E. 1963. Los tipos de vegetación de México y su clasificación. Boletín de la Sociedad Botánica de México 28:29-179.

Moya-Rodríguez J.G. 1982. Estudio descriptivo y florístico de las unidades sinecológicas de la Sierra La Silla, Nuevo León, México. Tesis de licenciatura, Facultad de Ciencias Biológicas, Universidad Autónoma de Nuevo León, San Nicolás de los Garza, N.L.83 pp.

Muller C.H. 1939. Relation of the vegetation and climatic types in Nuevo Leon, Mexico. American Midland Naturalist 21:687729.

Muller C.H. 1947. Vegetation and climate in Coahuila, Mexico. Madroño 9:33-57. 
Mueller-Dumbois D. y Ellenberg H. 1974. Aims and Methods of Vegetation Ecology. John Wiley \& Sons, Inc., Nueva York.

Panti-Madero M.A. 1992. Una comparación de la estructura de un matorral submontano en dos condiciones de explotación forestal. Tesis de Maestría, Universidad de Montemorelos, Montemorelos, N.L., México. 111 pp.

Pérez-García E.A. y Meave J.A. 2004. Heterogeneity of xerophytic vegetation of limestone outcrops in a tropical deciduous forest region in southern Mexico. Plant Ecology 175:147-163.

Ramírez-Álvarez E. 1984. Unidades fisonómico-florísticas de la Sierra Las Mitras, Nuevo León, México. Tesis de Licenciatura, Facultad de Ciencias Biológicas, Universidad Autónoma de Nuevo León, San Nicolás de los Garza, N.L., México. 76 pp.

Reid N., Marroquín J. y Beyer-Münzel P. 1990. Utilization of shrubs and trees for browse, fuel wood and timber in the tamaulipan thornscrub, northeast Mexico. Forest Ecology and Management 36:61-79.

Rojas-Mendoza P. 1965. Generalidades sobre la vegetación del estado de Nuevo León y datos acerca de su flora. Tesis de doctorado. Facultad de Ciencias, Universidad Nacional Autónoma de México, México, D.F. 124.

Rzedowski J. 1966. Vegetación del estado de San Luis Potosí. Acta Científica Potosina V. Universidad Autónoma de San Luis Potosí, San Luis Potosí.

Rzedowski J. 1978. Vegetación de México. Limusa, México, D.F.

Santibáñez-Andrade G., Castillo-Argüero S., Zavala-Hurtado J.A., Martínez-Orea Y. y Hernández-Apolinar M. 2009. La heterogeneidad ambiental en un matorral xerófilo. Boletín de la Sociedad Botánica de México 85:71-79.

Secretaría de Desarrollo Urbano y Obras Públicas. 2000. Áreas Naturales para la Conservación Ecológica en el Estado de Nuevo León. Secretaría de Desarrollo Urbano y Obras Públicas, Subsecretaría de Ecología. Monterrey, Nuevo León, México. 25 pp.

SEMARNAT [Secretaría del Medio ambiente y Recursos Naturales]. 2002. NORMA Oficial Mexicana NOM-059-ECOL-2001, Protección ambiental-Especies nativas de México de flora y fauna silvestres-Categorías de riesgo y especificaciones para su inclusión, exclusión o cambio-Lista de especies en riesgo. Diario Oficial de la Federación, 2aSección, 6 de marzo del 2002.

SEMARNAT. 2007. Plan de manejo tipo de venado cola blanca en climas áridos y semiáridos del norte de México. Secretaría del
Medio Ambiente y Recursos Naturales. México, D.F.

Sokal R.R. y Rohlf F.J. 1962. The comparison of dendrograms by objective methods. Taxon 11:33-40.

Steel R.G.D. y Torrie J.H. 1985. Bioestadística: Principios y Procedimientos. McGraw-Hill, México, D.F.

Torres W., Méndez M., Dorantes A. y Durán R. 2010. Estructura, composición y diversidad del matorral de duna costera en el litoral yucateco. Boletín de la Sociedad Botánica de México 86:37-51.

Trejo I. y Dirzo R. 2002. Floristic diversity of Mexican seasonally dry tropical forest. Biodiversity and Conservation 11:20632084.

Trejo-Vázquez I. 1999. El clima de la selva baja caducifolia en México. Investigaciones Geográficas (Mx) 39:40-52.

Villarreal-Quintanilla J.A. 1979. Vegetación del municipio de Los Ramones, Nuevo León. Tesis Licenciatura, Facultad de Ciencias Biológicas, Universidad Autónoma de Nuevo León, San Nicolás de los Garza, N.L., México. 68 pp.

Villarreal-Quintanilla J.A. y Estrada-Castillón A.E. 2008 Flora de Nuevo León. Listados Florísticos de México XXIV. Instituto de Biología, Universidad Nacional Autónoma de México, México, D.F.

Villaseñor J.L. 2004. Los géneros de plantas vasculares de la flora de México. Boletín de la Sociedad Botánica de México 75:105135.

Villegas-Durán G. 1972. Tipos de vegetación en los municipios de Linares y Hualahuises, N.L.; sus características, aprovechamiento y condiciones ecológicas en que se desarrollan. Tesis de licenciatura. Escuela de Agricultura. Universidad de Guadalajara, Guadalajara, Jalisco. 97 pp.

Ward J.H. 1963. Hierarchical grouping to optimize an objective function. Journal of the American Statistical Association 58:236-244.

White S.S. 1940.Vegetation of the Cerro de la Silla, near Monterrey, Mexico. Papers of the Michigan Academy of Science, Arts, and Letters 46:87-98.

Woerner P.M. 1991. Los suelos bajo vegetación del matorral del noreste de México, descritos a través de ejemplos en el campus universitario de la Universidad Autónoma de Nuevo León, Linares, Nuevo León. Reporte científico No 22. Facultad de Ciencias Forestales, Universidad Autónoma de Nuevo León, Linares. 
ClasificaCión, ESTRUCTURA Y DIVERSIDAD DEL MATORRAL SUBMONTANO

Apéndice 1. Especies de plantas vasculares registradas en los 39 sitios de muestreo en el matorral submontano y su forma de crecimiento (modificada de Font Quer 1977): Árbol (A), arbusto bajo (ab), arbusto medio (am), arbusto alto (al), inerme (i), espinoso (e), enredadera (en), parásita (p), suculenta (s) y rosetófila (r), áfila (af), caducifolio (ca), perennifolio (pe).

\section{PINOPHYTA}

\section{CUPRESSACEAE}

Juniperus pinchotii Sudw. [A]

EPHEDRACEAE

Ephedra pedunculata Engelm.ex S.Watson [ab/i/af/ca] PINACEAE

Pinus cembroides Zucc. [A]

TAXODIACEAE

Taxodium mucronatum Ten. [A]

\section{MANGOLIOPHYTA}

\section{LILIOPSIDA (MONOCOTILEDÓNEAS)}

AGAVACEAE

Agave albopilosa I.Cabral, Villarreal \& A.E.Estrada[r]

Agave bracteosa S.Watson ex Engelm. [r]

Agave lecheguilla Torr. [r]

Agave scabra Ortega var. scabra [r]

Agave striata Zucc. var. falcata (Engelm.) Gentry[r]

Agave victoriae-reginae T.Moore[r]

Dasylirionberlandieri S.Watson[r]

Dasylirion texanum Scheele[r]

Hesperaloe funifera (K.Koch) Trel. var. funifera [r]

Hesperaloe parviflora J.M.Coult.[r]

Yucca carnerosana (Trel.) McKelvey[r]

Yucca filifera Chabaud[r]

Yucca rostrata Engelm. [r]

Yucca torreyi Shafer var. torreyi [r]

Yucca treculeana Carrière[r]

ARECACEAE

Brahea berlandieri Bartlett[r]

Sabal mexicana Mart. [r]

SMILACACEAE

Smilax bona-nox L. [en]

\section{MAGNOLIOPSIDA (DICOTILEDÓNEAS)}

ACANTHACEAE

Anisacanthus quadrifidus Standl. var. wrightii (Torr.)

Hernrickson[am/i/ca]

Carlowrightiatorreyana Wassh. [h]

ANACARDIACEAE

Pistacia mexicana Kunth [am/i/pe]

Rhus microphylla Engelm. [am/i/pe]

Rhus pachyrrhachis Hemsl. [am/i/pe]

Rhus virens Lindh. ex A.Gray var. virens [am/i/pe]

Toxicodendron radicans Kuntze var. eximium (Greene)

F.A.Barkley [en]

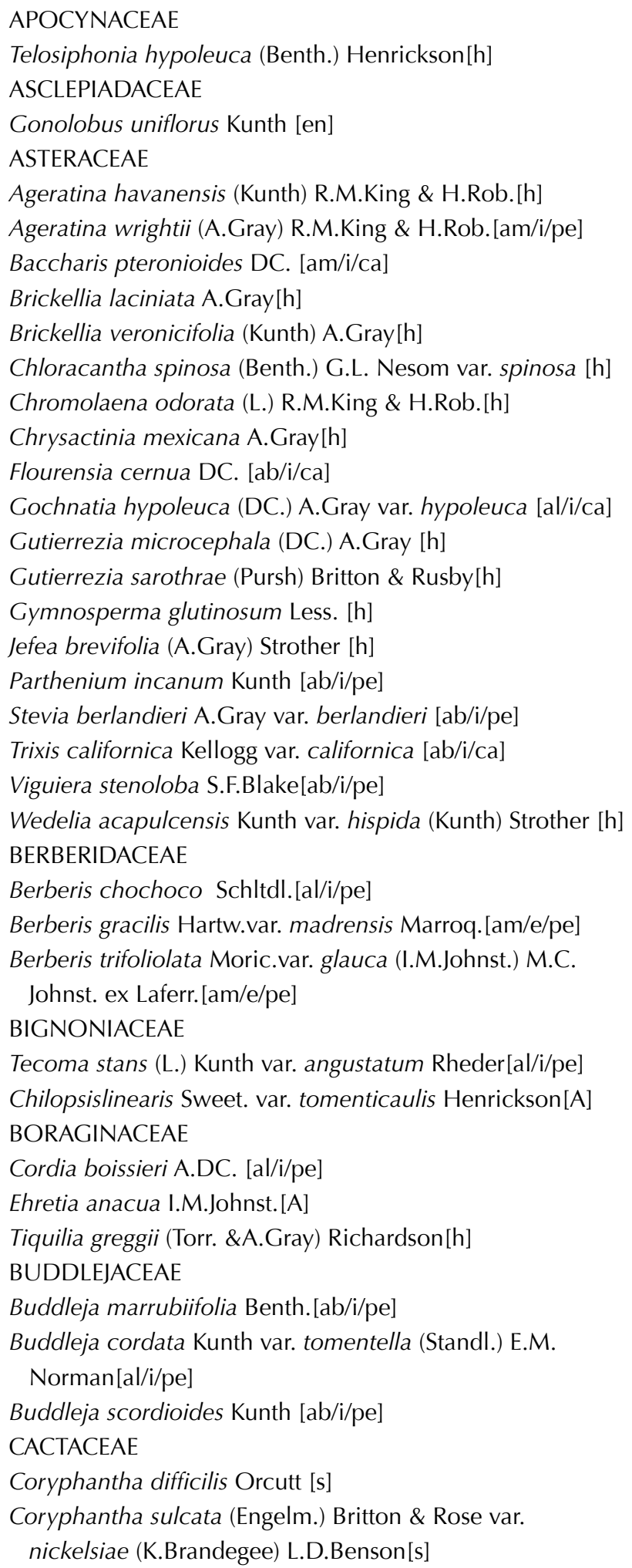


Coryphantha sulcata (Engelm.) Britton \& Rose. var. sulcata [s] Echinocactus texensis Hopffer[s]

Echinocereus blanki F.Palmer[s]

Echinocereus enneacanthus Engelm. var. enneacanthus[s]

Echinocereus pectinatus Engelm. [s]

Echinocerus poselgeri Lem. [s]

Echinocereus reichenbachii (Terscheck ex Walp.) Britton \&

Rose var. reichenbachii [s]

Echinocereus viereckii Werderm. var. huastecensis W.Blum,

Mich.Lange \& Rutow[s]

Escobaria emskoetteriana (Quehl) Borg[s]

Escobaria tuberculosa Britton \& Rose[s]

Ferocactus haematacanthus (Salm-Dyck) Borg ex Backeb. [s] Mammillaria heyderi Muehlenpf. var. gummifera (Engelm.)

D.R.Hunt [s]

Mammillaria heyderi Muehlenpf. var. hemisphaerica

Engelm. [s]

Mammillaria heyderi Muehlenpf. var. heyderi[s]

Mammillaria melanocentra Poselg. [s]

Mammillaria plumosa F.A.C.Weber [s]

Mammillaria prolifera (Mill.) Haw. [s]

Opuntia engelmannii Salm-Dyck ex Engelm. [s]

Opuntia leptocaulis DC. [s]

Opuntia macrocentra Engelm. [s]

Opuntia phaeacantha Engelm. [s]

Opuntia tunicata (Lehm.) Pfeiff. [s]

Sclerocactus scheeri (Salm-Dyck) N.P.Taylor[s]

Thelocactus bicolor (Galotii ex Pfeiff.) Britton \& Rose [s]

CAPPARACEAE

Koeberlinia spinosa Zucc. [am/e/af]

CELASTRACEAE

Schaefferia cuneifolia A.Gray[ab/i/ca]

EBENACEAE

Diospyros palmeri Eastw. [A]

Diospyros texana Scheele [A]

ERICACEAE

Arbutus xalapensis Kunth [A]

Arctostaphylos pungens Kunth [am/i/pe]

EUPHORBIACEAE

Bernardia myricifolia (Scheele) S.Watson var. myricifolia

[am/i/ca]

Croton ciliatog/andulifer Ortega [ab/i/ca]

Croton cortesianus Kunth [ab/i/ca]

Croton incanus Kunth [ab/i/ca]

Croton fruticulosus Torr. [ab/i/ca]

Croton suaveolens Torr. [am/i/ca]

Euphorbia antisyphilitica J.Meyrán[ab/i/af]

Jatropha dioica Sessé var. graminea McVaugh [ab/i/ca]

\section{FAGACEAE}

Quercus canbyi Trel. [A]

Quercus fusiformis Small [A]

Querus intricata Trel. [A]

Quercus laceyi Small [A]

Quercus polymorpha Schltdl. \& Cham. [A]

Quercus pungens Liebm. var. vaseyana (Buckley)

A.E.Murray $[\mathrm{A}]$

Quercus rysophylla Weath $[\mathrm{A}]$

Quercus sinuata Walter var. breviloba (Torr.) A.E.Murray. [A]

FLACOURTIACEAE

Xylosma flexuosa (Kunth) Hemsl. [am/e/ca]

HYDRANGEACEAE

Philadelphus microphyllus A.Gray[am/i/ca]

JUGLANDACEAE

Carya myristiciformis (F.Michx.) Elliot[A]

Carya ovata (Mill.) K.Koch var. mexicana (Engelm. ex Hemsl.)

W.E.Manning $[\mathrm{A}]$

Juglans mollis Engelm. [A]

KRAMERIACEAE

Krameria grayi Rose \&J.H.Painter[ab/i/ca]

Krameria ramosissima (A.Gray) S.Watson [ab/i/ca]

LAMIACEAE

Salvia ballotiflora Benth. [am/i/ca]

LAURACEAE

Litsea pringlei Bartlett [am/i/ca]

LEGUMINOSAE

Acacia amentacea DC. [al/e/ca]

Acacia berlandieri Benth. [am/i/ca]

Acacia coulteri Benth. [al/i/ca]

Acacia farnesiana (L.) Willd. [A]

Acacia greggii A.Gray var. greggii [al/e/ca]

Acacia roemeriana Scheele [am/e/ca]

Acacia schaffneri (S. Watson) F.J.Herm. var. bravoensis

Isely [al/e/ca]

Acaciella angustissima (Mill.) Britton \& Rose var.

angustissima $[\mathrm{am} / \mathrm{i} / \mathrm{ca}]$

Bauhinia macranthera Benth. ex Hemsl. [al/i/ca]

Bauhinia lunarioides A.Gray ex S.Watson [am/i/ca]

Bauhinia ramosissima Benth. ex Hemsl. var. uniflora

(S Watson) M.P.Ramírez \&R.Torres[ab/i/ca]

Caesalpinia mexicana A.Gray [al/i/ca]

Calia secundiflora (Ortega) Yakovlev [al/i/pe]

Calliandra conferta Benth. [ab/i/ca]

Canavalia villosa Benth. [en]

Cercis canadensis L. var. mexicana (Rose) M.Hopkins [A]

Chamaecrista greggii (A.Gray) Pollard ex A.Heller var.

greggii [ab/i/pe] 
Dalea frutescens A.Gray [ab/i/ca]

Dalea lutea Willd. var. lutea [ab/i/ca]

Dalea melantha S.Schauer var. melantha [am/i/ca]

Dalea scandens (Mill.) R.T.Clausen var. paucifolia

(J.M.Coult.) Barneby [ab/i/ca]

Ebenopsis ebano (Berland.) Barneby \& J.W.Grimes [A]

Erythrina flabelliformis Kearney [al/e/ca]

Eysenhardtia texana Scheele [al/i/ca]

Havardia pallens Britton \& Rose [al/e/ca]

Leucaena greggii S.Watson [al/i/ca]

Leucaena leucocephala (Lam.) deWit var. glabrata

(Rose) Zárate [A]

Leucaena pulverulenta Benth. [A]

Mimosa aculeaticarpa Ortega [am/e/ca]

Myrospermum sousanum Delgado \& M.C.Johnston [A]

Oxyrhynchus volubilis Brandegee [en]

Parkinsonia aculeata L. [A]

Parkinsonia texana S.Watson var. macra (I.M.Johnst.) Isely [A]

Parkinsonia texana S.Watson var. texana [am/e/ca]

Prosopis laevigata (Humb. \&Bonpl. ex Willd.)

M.C.Johnston [A]

Prosopis glandulosa Torr. var. torreyana (L.D.Benson)

M.C.Johnston [A]

Tephrosia potosina Brandegee [h]

LYTHRACEAE

Heimia salicifolia Link \& Otto [am/i/pe]

MALPIGHIACEAE

Aspicarpa hyssopifolia A.Gray [ab/i/pe]

Mascagnia lilacina (S.Watson) Nied. [ab/i/ca]

Mascagnia macroptera Nied. [ab/i/ca]

MALVACEAE

Abutilon glabriflorum Hochr. [h]

Abutilon hypoleucum A.Gray[h]

Abutilon malacum S.Watson[h]

Hibiscus acicularis Standl. [h]

Hibiscus martianus Zucc. [h]

Meximalva filipes (A.Gray) Fryxell [h]

Pavonia lasiopetala Scheele [h]

MELIACEAE

Melia azedarach L. [A]

OLEACEAE

Forestiera angustifolia Torr. [am/i/ca]

Forestiera reticulata Torr. [am/i/ca]

Fraxinus greggii A.Gray var. greggii $[\mathrm{A}]$

Menodora longiflora A.Gray [h]

PHYTOLACCACEAE

Phytolacca icosandra L. [h]

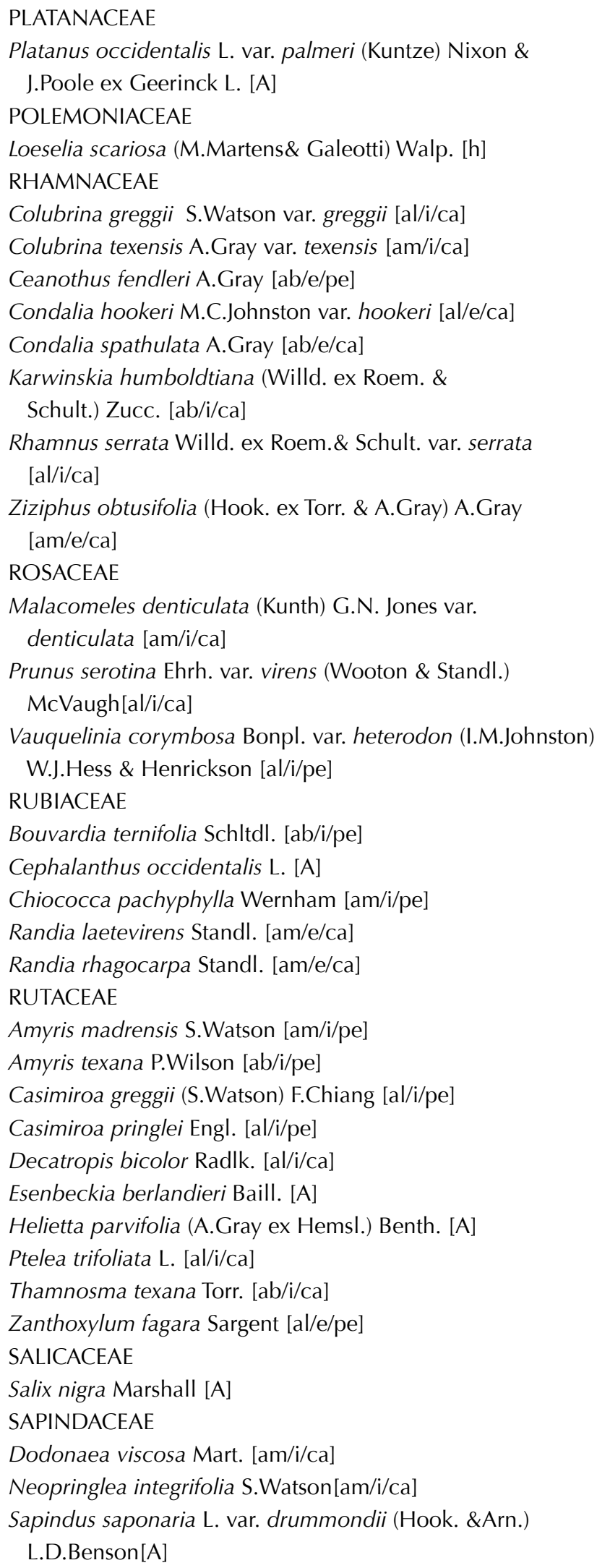


Serjania brachycarpa A.Gray [en]

Ungnadia speciosa Endl. [al/i/ca]

Urvillea ulmacea Kunth [en]

SAPOTACEAE

Sideroxylon lanuginosum Michx. var. rigidum (A.Gray)

B.L.Turner [al/e/ca]

Sideroxylon celastrinum (Kunth) T.D.Penn. [al/e/ca]

SCROPHULARIACEAE

Leucophyllum frutescens I.M.Johnst. [ab/i/pe]

SIMAROUBACEAE

Castela erecta Turpin var. texana (Torr. \& A.Gray)

Cronquist [ab/i/ca]

SOLANACEAE

Capsicum annum L.var. glabriusculum (Dunal) C.B.Heiser \& Pickersgill [am/i/ca]

Lycium berlandieri Dunal var. berlandieri [am/e/ca]

Nicotiana glauca Graham [al/i/ca]

Solanum erianthum D. Don. [am/i/ca]

STERCULIACEAE

Melochia pyramidata L. [ab/i/ca]

Waltheria indica L. [ab/i/ca]

TURNERACEAE

Turnera diffusa Willd. ex Schult. [ab/i/ca]

\section{ULMACEAE}

Celtis laevigata Willd. [A]

Celtis pallida Torr. [am/e/ca]

Celtis reticulata Torr. [al/i/ca]

Ulmus crassifolia Nutt. [A]

VERBENACEAE

Aloysia gratissima (Gillies \& Hook.) Tronc. var. gratissima [am/i/ca]

Aloysia macrostachya (Torr.) Moldenke [am/i/ca]

Citharexylum berlandieri B.L.Rob. [am/i/ca]

Citharexylum brachyanthum (A.Gray ex Hemsl.) A.Gray var. brachyanthum [am/i/ca]

Lantana camara L. [ab/i/pe]

Lantana macropoda Torr. [ab/i/ca]

Lippia graveolens Kunth [am/i/ca]

VISCACEAE

Arceuthobium vaginatum J.Presl. [p]

Phoradendron tomentosum (DC.) A.Gray [p]

VITACEAE

Parthenocissus quinquefolia (L.) Planch [en]

Vitis berlandieri Planch [en]

ZYGOPHYLLACEAE

Guaiacum angustifolium Engelm. [ab/i/pe] 